\title{
Micro-Impact Damage Caused by Mercury Bubble Collapse*
}

\author{
Masatoshi FUTAKAWA**, Takashi NAOE***, Hiroyuki KOGAWA**, \\ Hidefumi DATE $^{* * * *}$ and Yujiro IKEDA**
}

\begin{abstract}
High-power spallation targets for neutron sources are being developed in the world. Mercury target will be installed at the material science and life facility in J-PARC, which will promote innovative science. The mercury target will be subjected to the pressure wave caused by proton bombarding in the mercury. The pressure wave propagation induces the cavitation in mercury that imposes localized impact damage on the target vessel. The impact erosion is a critical issue to decide the lifetime of target. An electromagnetic impact testing machine, MIMTM, was developed to reproduce the localized impact erosion damage and evaluate the damage formation. Additionally, droplet impact analyses were carried out to investigate the correlation between isolate pit profile and micro-jet velocity. We confirmed that the value of depth/radius was applicable to estimate micro-jet velocity, and the velocity at $560 \mathrm{~W}$ in MIMTM equivalent to $1 \mathrm{MW}$ proton beam injection was $\sim 10^{2} \mathrm{~m} / \mathrm{s}$ approximately.
\end{abstract}

Key Words: Spallation Target, Mercury, Cavitation Erosion, Pitting Damage, Droplet, Impact Analysis

\section{Introduction}

A MW-class target that is planned to be constructed in the world can produce a high-intensity neutron beam by the spallation reaction due to accelerated protons bombarding at the target materials. A liquid-mercury target system for the MW-class target is being developed in the JAERI as taking into account the neutron yield and selfcirculating heat removal efficiencies, and will be installed at the JSNS (Japan Spallation Neutron Source) in J-PARC (Japan Proton Accelerator Research Complex) $^{(1)}$. The proton beam with a $1 \mu$ s pulse duration is injected into the mercury through a beam window at $25 \mathrm{~Hz}$. The moment the proton beams bombard the target, stress waves will be imposed on the beam window and pressure waves will be generated in the mercury by thermally shocked heat deposition. Provided that pressure waves impose impact forces on the interface between liquid/solid metals and the negative pressure generates through the pressure wave propagation in the mercury target, there is the possibility for

\footnotetext{
* Received 20th May, 2005 (No. 05-4110)

** Japan Atomic Energy Research Institute, Toukai-mura, Naka-gun, Ibaraki 319-1112, Japan.

E-mail: futakawa@popsvr.tokai.jaeri.go.jp

*** Ibaraki University, Hitachi-shi, Ibaraki 316-8511, Japan

**** Tohoku Gakuin University, 1-13-1 Chuo, Tagajo 9858537, Japan
}

formation of cavitation in the mercury and the cavitation micro-bubble collapse to form pits on the interface under the proton injection. Off-line tests on the pressure waves without proton beams had been carried out to evaluate the dynamic response of mercury by using SHPB (Split Hopkinson Pressure Bar) impact machine. We found the pitting damage on the interface between solid metal and mercury $^{(2),(3)}$. Following the SHPB tests, the on-beam tests using proton beams had been performed to confirm the pitting damage observed in the off-line tests ${ }^{(4)}$. After both the experimental results, the so-called pitting damage becomes a crucial issue to realize high power spallation neutron sources.

In order to systematically examine the pitting damage on the target vessel under certain conditions, the offline tests were performed by using an innovative device, MIMTM (electroMagnetic IMpact Testing Machine) to simulate the pressure waves due to proton bombarding mercury $^{(5)}$. In this paper, the relationship between the localized impact force to form the pits and the morphology of damaged surface with crater holes of pits will be discussed through the comparison between the experimental and calculated results by FEM code, in order to understand precisely the relationship between the pitting damage and the beam power and to improve the surface properties to prevent from the pitting damage. 


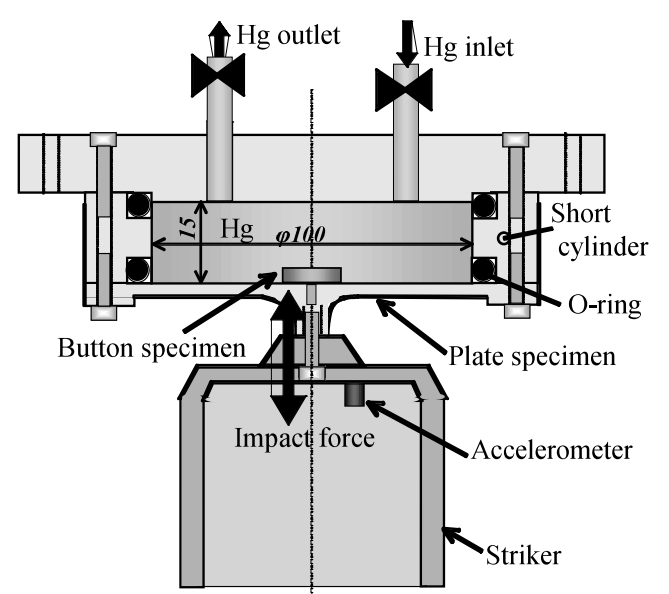

Fig. 1 Mercury chamber and specimen in MIMTM

Table 1 Chemical composites of $316 \mathrm{ss}$ (wt $\%$ )

\begin{tabular}{|c|c|c|c|c|c|c|c|c|}
\hline $\mathrm{C}$ & $\mathrm{Si}$ & $\mathrm{Mn}$ & $\mathrm{P}$ & $\mathrm{S}$ & $\mathrm{Ni}$ & $\mathrm{Cr}$ & $\mathrm{Mo}$ & $\mathrm{Fe}$ \\
\hline 0.05 & 0.49 & 1.30 & 0.03 & 0.02 & 10.3 & 16.9 & 2.07 & Bal. \\
\hline
\end{tabular}

\section{Experiment}

Figure 1 shows the mercury chamber and specimen in the MIMTM. The inner diameter and height are $\phi 100 \mathrm{~mm}$ and $15 \mathrm{~mm}$, respectively. The button-type specimen with $\phi 15 \mathrm{~mm}$ diameter is fixed at the center of the disk plate. The impulsive pressure is imposed to the mercury through the disk plate driven with the striker controlled by the electromagnetic force. The magnitude of pressure is varied by the imposed power into the MIMTM. The morphology of pitting damage observed at the power of $560 \mathrm{~W}$ in the MIMTM is sufficiently equivalent to that in the onbeam tests using MW-class proton beams ${ }^{(5)}$. The repeated frequency of pulses is $25 \mathrm{~Hz}$ which is the same as that in JSNS.

The materials of specimen are austenitic stainless steel type 316 and surface improved 316ss with nitriding and carburizing treatments. Table 1 shows the chemical compositions of 316 ss. The tests on power and materials were carried out under the following conditions: 1) power dependency; the number of pulses is $10^{3}$ constant, imposed power is varied in the range from $185 \mathrm{~W}$ to $690 \mathrm{~W}$, and the material is $316 \mathrm{ss}, 2$ ) material dependency; the imposed power is $560 \mathrm{~W}$ constant and the number of pulses is $10^{4}$ constant to distinguish the differences in isolated pit morphology of various materials, 316ss and surface improved ones. The morphology and depth profile of pits are observed using a laser microscope.

\section{Impact Analysis}

The pits are formed by the micro-bubble collapses that impose the localized impact on the solid/liquid interface by the micro-jets and/or shock waves collision against it. As assuming that the micro-jets are the most effective

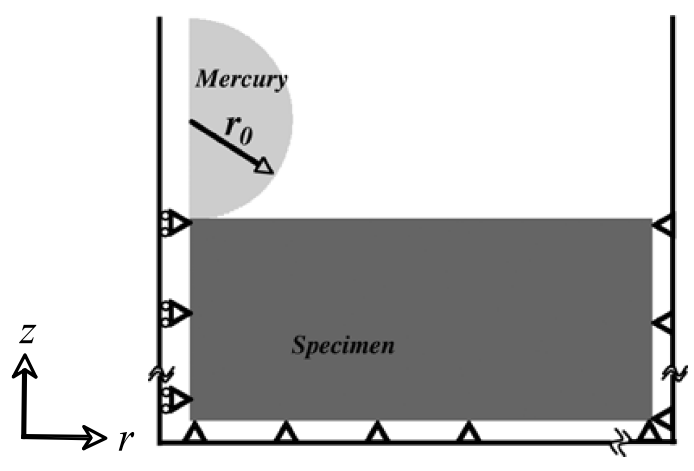

Fig. 2 Impact analytical model of solid/liquid interface

to form the pits, the analyses were carried out using an axisymmetrical model consisting of a spherical mercury droplet and a flat solid plate, as shown in Fig. 2. The mercury droplet was meshed in Eulerian coordinate and the solid plate in Lagurangian to be taken a large deformation into account by using AUTODYN-2D code ${ }^{(6)}$. The boundary conditions in the solid plate are free along to the collision surface and perfectly fixed conditions at the other ones. The following constitutive equations are used:

1) For the droplet of mercury

Rankine-Hugoniot equation was applied to estimate the imposed pressure on the interface as follow;

$$
p=p_{H}+\Gamma \rho\left(E-E_{H}\right),
$$

where $\Gamma$ is Mie-Gruneisen coefficient, $E$ internal energy, subscript $H$ the value of Hugoniot-curve. Assuming that the internal energy is hardly varied by impact, the second term was ignored. $p_{H}$ is given by

$$
p_{H}=\frac{\rho_{0} c_{0} 2 \mu(1+\mu)}{[1-(s-1) \mu]^{2}}, \quad \mu=\frac{\rho-\rho_{0}}{\rho},
$$

where $c_{0}$ is sound velocity, $\rho_{0}$ initial density and $s$ shock wave velocity parameter. For the mercury, $c_{0}=1490 \mathrm{~m} / \mathrm{s}$, $\rho_{0}=13.54 \times 10^{3} \mathrm{~kg} / \mathrm{m}^{3}, s=2.047$, and $\Gamma=1.96^{(7)}$.

2) For the solid plate

Johnson-Cook equation was applied to take the strain rate effect into account. Dynamic flow stress $\sigma_{y d}$ is given by

$$
\begin{aligned}
\sigma_{y_{d}} & =\left[A+B \varepsilon_{p}^{n}\right]\left[1+C \log \dot{\varepsilon}_{p}\right]\left[1-T_{H}^{m}\right], \\
T_{H} & =\left(T-T_{R}\right) /\left(T_{m}-T_{R}\right)
\end{aligned}
$$

where $\varepsilon_{p}$ is plastic strain, $\dot{\varepsilon}_{p}$ plastic strain rate, $T$ temperature, $T_{R}$ room temperature, $T_{m}$ melting temperature, $A$ static yield stress, $B$ work hardening coefficient and $n$ work hardening exponent, $C$ strain rate work hardening coefficient, $m$ softening coefficient. Temperature dependency is ignored. The depth profilet of pre-analysis with $C=0 \sim 10^{2}$ were hardly changed. Thus, as ignoring the strain rate effect, Eq. (3) becomes bi-linear equation. In the analysis, $A=203 \mathrm{MPa}, B=958 \mathrm{MPa}, n=1, C=0$ and $m=1$ for $316 \mathrm{ss}^{(8)}$. The material properties; $A$ and $B$ for the carburizing and nitriding treated surface layers were estimated by using the inverse analysis on the load/depth curves measured by nanoindentation technique that was 
developed by the authors ${ }^{(9)}$. That is, $A=1615 \mathrm{MPa}$ and $B=1157 \mathrm{MPa}$ for the carburizing layer and $A=2247 \mathrm{MPa}$ and $B=1390 \mathrm{MPa}$ for the nitriding. The values of $n, C$ and $m$ are assumed to be the same as those in the substrate of 316 ss.

\section{Results}

\section{1 Morphology of pit}

Figure 3 shows the power dependency on the micrographs of pits in 316ss. The isolated pits are observed. The pit shape becomes obvious as increasing the power. Any pits were not observed in the range lower than $185 \mathrm{~W}$. Figure 4 shows the depth profiles of pits. Both the diameter and depth of pits increase with the increase of power. Figure 5 shows the relationship between power and shape of pit as depth/radius, $D / R$ that is; $D$ is the maximum depth and $R$ the radius of equivalent circle in deformed area. It is seen that the threshold of pit formation to the power is present, i.e. $200 \mathrm{~W}$ approximately, and $D / R$ increases with power independently of pit size itself.

Figures 6 and 7 show the material dependencies on the micrograph and on the depth profiles of pits in 316ss substrate, and carburizing and nitriding surface layers. The pit of nitriding was hardly observed even after $10^{4}$ pulses, and then the pit of nitriding at $10^{6}$ pulses was com-

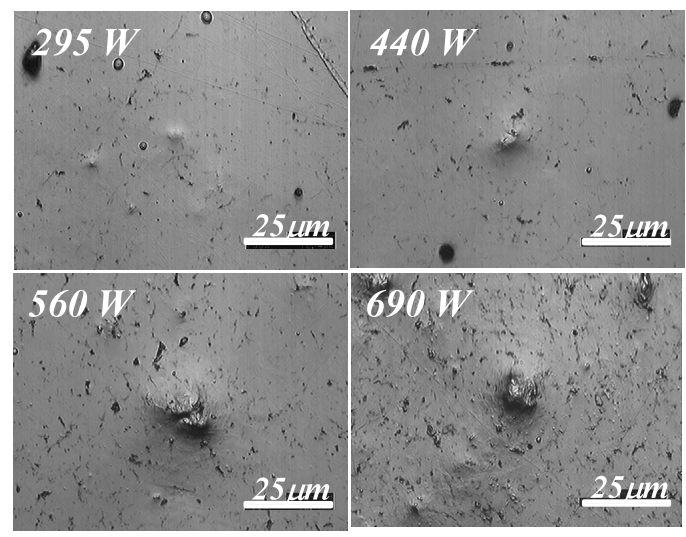

Fig. 3 Power dependency on micrograph in 316ss

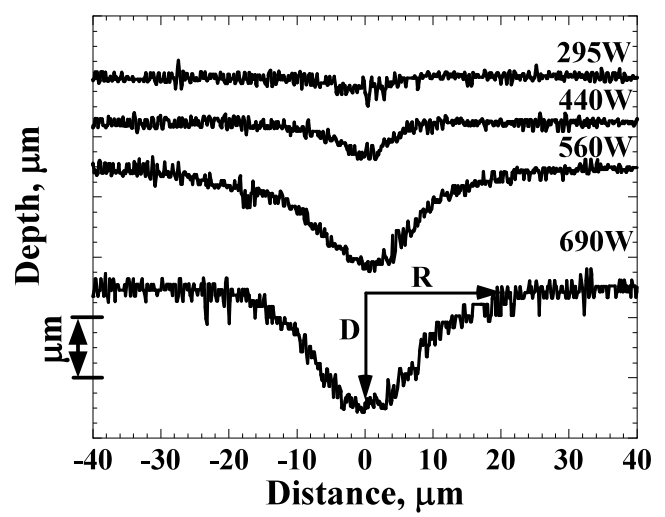

Fig. 4 Power dependency on depth profile of pit in 316ss pared with those of $316 \mathrm{ss}$ and carburizing at $10^{4}$ pulses. As compared with the depth profiles of 316 ss and carburizing, the nitriding exhibits a sharp depth change. The plastic deformation might be not expected in the nitriding surface layer unlike 316ss and carburizing, since the slip lines are recognized in the 316 ss and carburizing whereas not in the nitriding.

\subsection{Effect of impact velocity on depth profile of pit}

Figure 8 shows analytical results of depth profiles of pits with various impact velocities in the case of 316 ss. The depth of pit $z$ and the distance from the center of pit $r$ are normalized by the radius $r_{0}$ of mercury droplet, respectively. The impact velocity dependency is much more sensitively enhanced in the depth rather than in the radius. The pile-up due to large plastic deformation gets to be distinguished in the range more than $225 \mathrm{~m} / \mathrm{s}$.

Figures 9 and 10 show the analyzed depth profiles of pits formed on the carburizing and the nitriding surface layers. The depths of pits in the surface layers are much shallower than those in the 316 ss even in the range up to

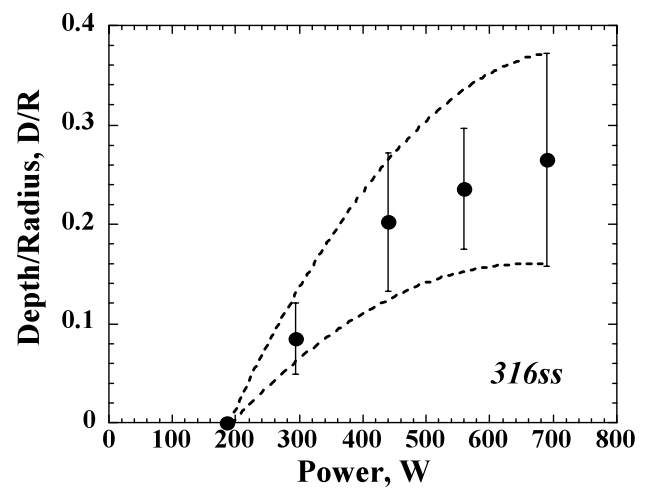

Fig. 5 Relationship between power and $D / R$ of pit

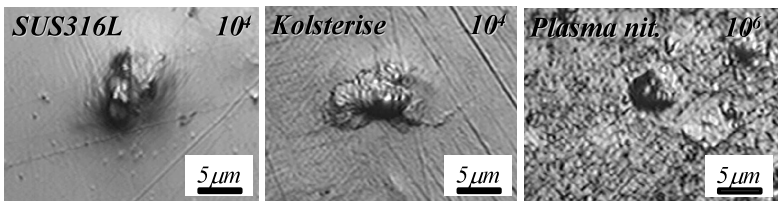

Fig. 6 Material dependency on micrograph in 316ss and carburizing and nitriding treated surface layers

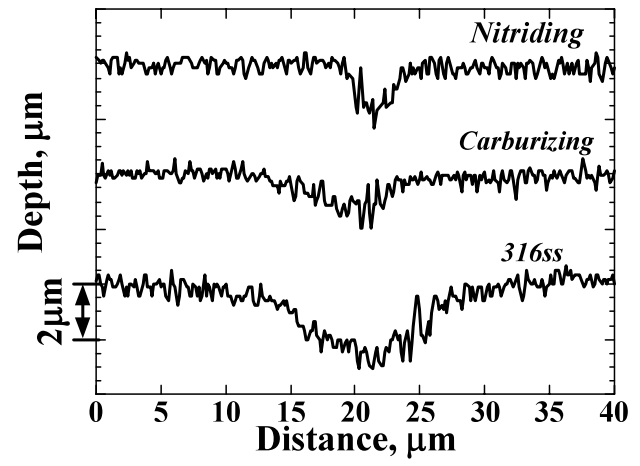

Fig. 7 Material dependency on depth profiles of pits 


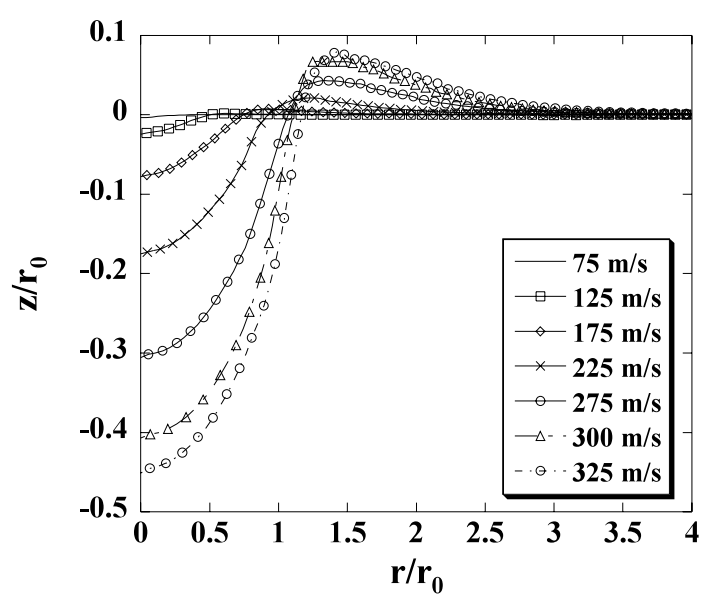

Fig. 8 Analyzed depth profiles of pits under various impact velocities in 316 ss

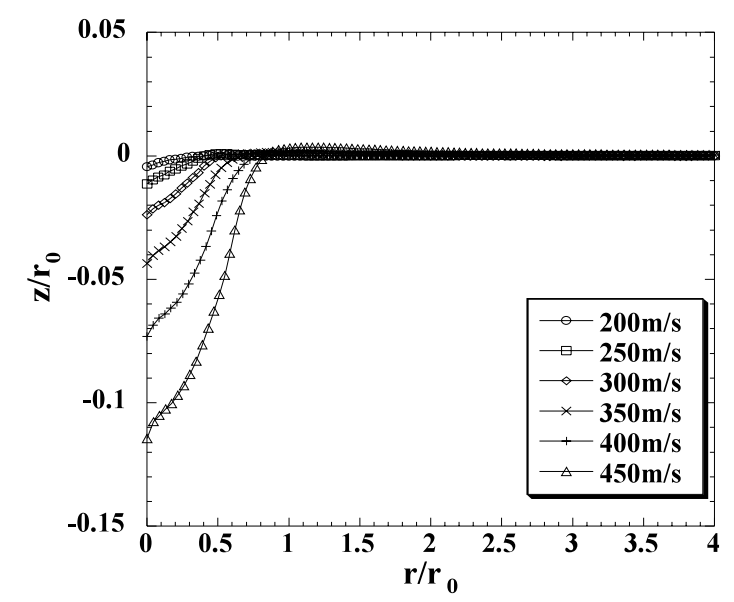

Fig. 9 Analyzed depth profiles of pits under various impact velocities in carburizing treated surface layer

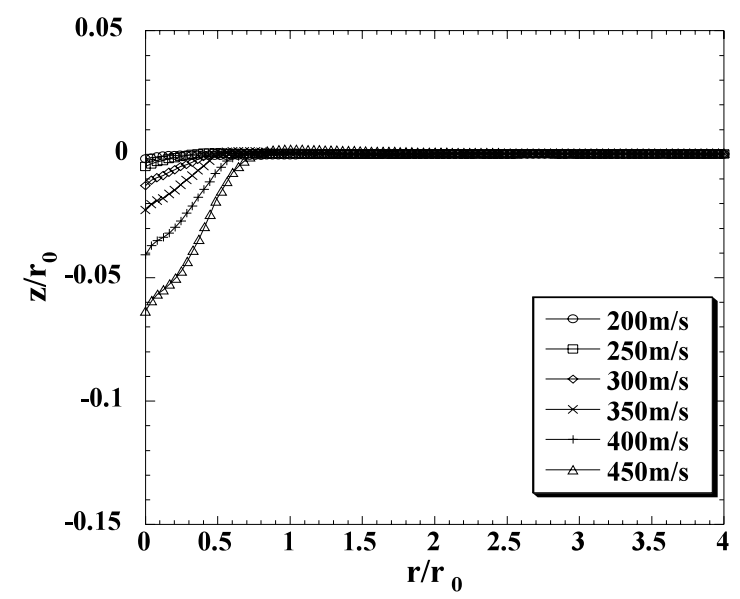

Fig. 10 Analyzed depth profiles of pits under various impact velocities in nitriding treated surface layer

$450 \mathrm{~m} / \mathrm{s}$. The pile-up is little in the nitriding.

Figure 11 shows the relationship between impact velocity and $D / R$ of pits. The threshold of pit formation to the impact velocity is present because the impact energy

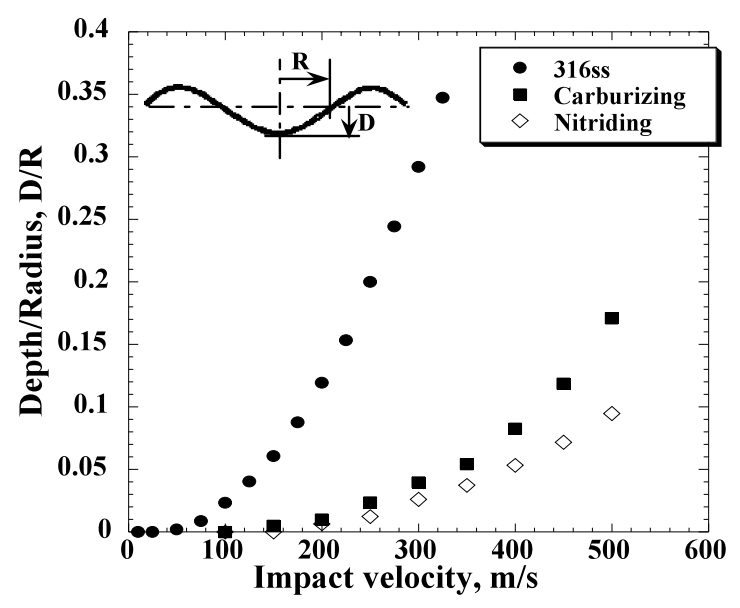

Fig. 11 Relationship between impact velocity and $D / R$ of pits

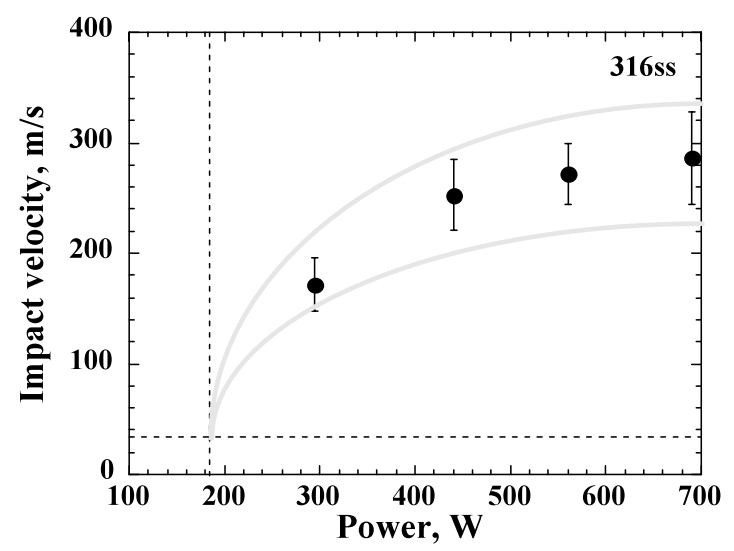

Fig. 12 Relationship between impact velocity and power

is dispersed by elastic deformation in the range lower than $50 \mathrm{~m} / \mathrm{s}$ in the $316 \mathrm{ss}$ and $150 \mathrm{~m} / \mathrm{s}$ in the carburizing and nitriding, approximately. It is found that the impact velocity of micro-jet driven by the micro-bubble collapse can be deduced from the pit depth profile, i.e. $D / R$.

\section{Discussion}

\subsection{Relationship between power and impact veloc- ity}

It was found from experimental and analytical results on the morphology of pits that the $D / R$ gives the information on the imposed power as well as the impact velocity of micro-jet. The relationship between impact velocity and power in $316 \mathrm{ss}$ is given in Fig. 12 by taking account of the results shown in Figs. 5 and 11. It is confirmed that the micro-jet with impact velocity more than $50 \mathrm{~m} / \mathrm{s}$, i.e. the threshold to the pit formation, is induced in the range more than the power of $200 \mathrm{~W}$ approximately, and that the impact velocity at $560 \mathrm{~W}$ which is equivalent to the power condition of MW-class proton beams ranges from 250 to $300 \mathrm{~m} / \mathrm{s}$.

Figure 13 illustrates the depth profiles of pits in 316ss that are obtained experimentally and analytically under the power condition at $560 \mathrm{~W}$ in MIMTM equivalent to MW- 


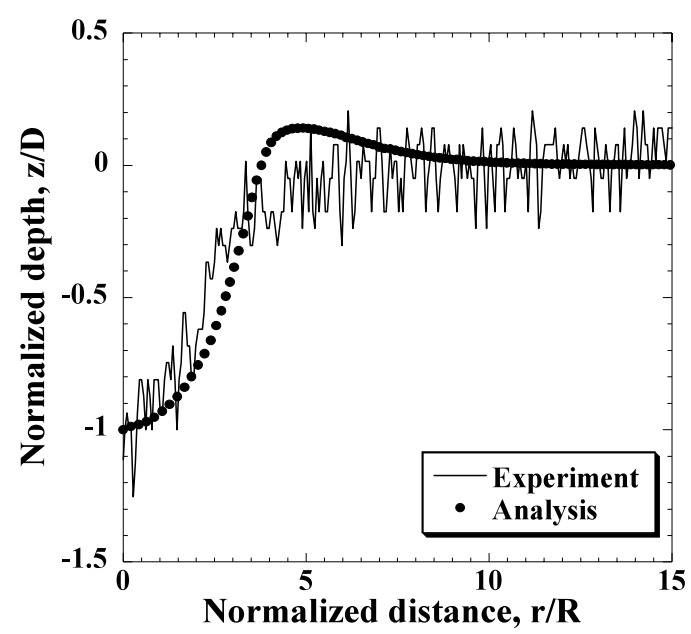

Fig. 13 Comparison of pit profile between experimental and analytical results of $316 \mathrm{ss}$ at $560 \mathrm{~W}$ in MIMTM equivalent to $\mathrm{MW}$-class proton beam power

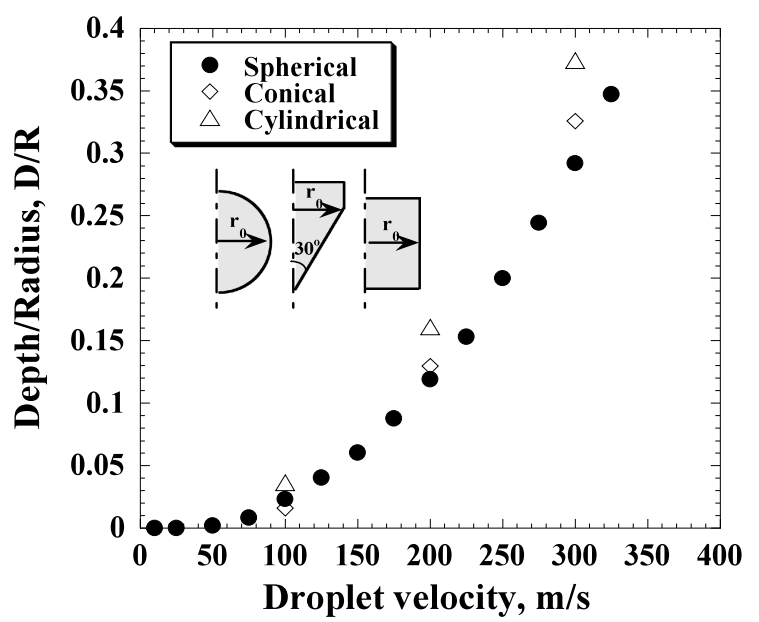

Fig. 14 Effect of droplet shape on pit depth profile

class proton beams. The analytical result gives a good agreement with the experimental one.

\subsection{Effect of droplet shape on pit profile}

Figure 14 shows the effect of the droplet shapes on the pit depth profiles; i.e. the relationship between $D / R$ and impact velocity with various shapes of droplets: spherical, cylindrical and conical shapes which have the same volume. The $D / R$ of pit is almost independent of the shape of droplet in the range up to $300 \mathrm{~m} / \mathrm{s}$. It is confirmed, therefore, that the $D / R$ is a unique parameter to estimate the impact velocity of micro-jet on the solid/liquid interface.

\subsection{Effect of thickness of improved surface layer on pit formation}

The pit formation may be affected by the interaction between the improved surface layer and the substrate. Figure 15 shows the effect of the thickness of the carburizing treated surface layer on the pit formation calculated at $300 \mathrm{~m} / \mathrm{s}$. The $D$ and $R$ of pit and the thickness $t$ are normalized by the radius $r_{0}$ of droplet. The depth of pit

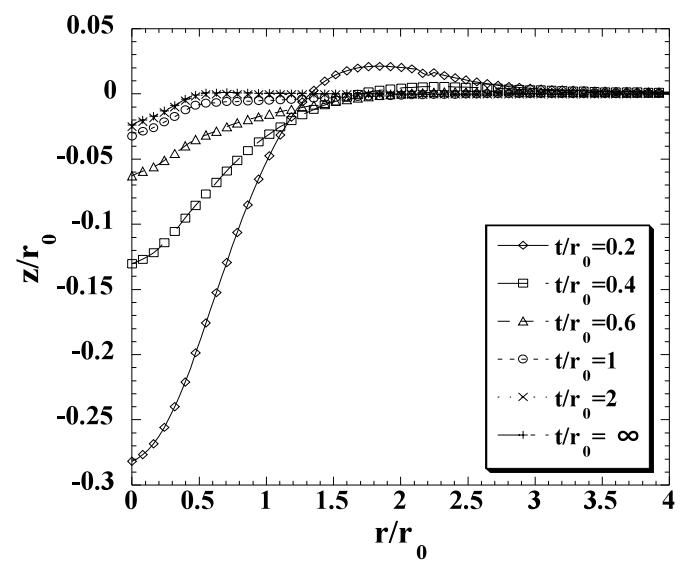

Fig. 15 Pit formation affected by carburizing treated surface layer

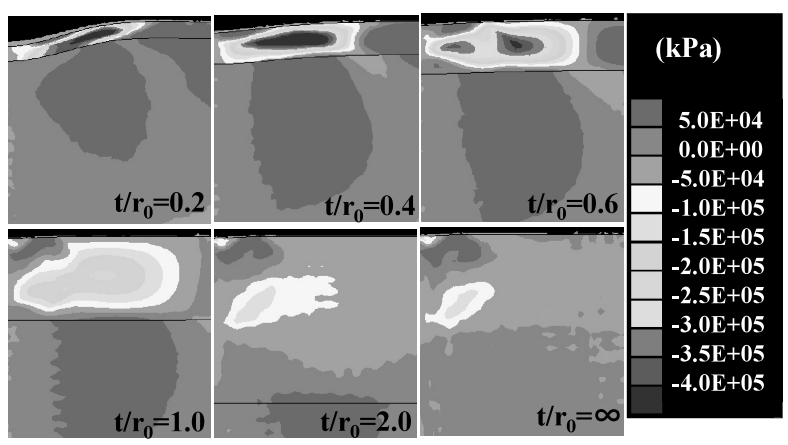

Fig. 16 Shear stress distribution along to the interface between carburizing treated surface layer and 316ss substrate at the impact velocity of $300 \mathrm{~m} / \mathrm{s}$

increases as decreasing the thickness of the carburizing surface layer. In the range of $t / r_{0}>1$, the depth profiles are not distinguished each other.

Figure 16 illustrates the shear stress distributions with the various values of $t / r_{0}$. Large shear stresses impose along to the interface between surface layer and substrate when $t / r_{0}<1$.

In the range of $t / r_{0}>2$, the shear stress distribution is not dependent on the thickness of the carburizing treated surface layer: i.e. the same as the stress distribution of homogeneously carburized 316 ss.

\section{Conclusion}

The localized impact phenomenon induced by microbubble collapse under the cavitation that is generated by the impulsive pressure was precisely examined through experiments and analyses. As results, it is confirmed that the value of radius/depth in pit is unique parameter to decide the localized impact velocity, and the impact velocity reaches to $300 \mathrm{~m} / \mathrm{s}$ approximately under the MW-class proton beam bombarding conditions.

This research is partly supported by a Grant-in-Aid in Scientific Research under the Japan Society for Promotion 
of Science, No.16206097 and No.17360085.

\section{References}

( 1 ) Planning Division for Neutron Science (1999), JAERIConf 99-003, (1999).

( 2 ) Futakawa, M., Kogawa, H. and Hino, R., Measurement of Dynamic Response of Liquid Metal Subjected to Uniaxial Strain Wave, J. Phys. IV France, 10 (2000), Pr9-237-242.

( 3 ) Futakawa, M., Kogawa, K., Hino, R., Date, H. and Takeishi, H., Erosion Damage on Solid Boundaries in Contact with Liquid Metals by Impulsive Pressure Injection, Int. J. Imp. Eng., 28 (2003), pp.123-135.

( 4 ) Haines, J.R., Farrell, K., Hunn, J.D., Lousteau, D.C., Mansur, L.K., McManamy, T.J., Pawel, S.J. and Riemer, B.W., Summary of Mercury Target Pitting Issue, SNS-101060100-TR0004, (2002).
( 5 ) Futakawa, M., Naoe, T., Kogawa, H., Tsai, C. and Ikeda, Y., Pitting Damage Formation up to over 10 Million Cycles-Off-Line Test by MIMTM-, J. Nucl. Sci. Tech., Vol.40, No.11 (2003), pp.895-904.

(6) Century Dynamics Inc., AUTODYN Theory Manual, Ver.4.1, (2000).

( 7 ) Group GMX-6, Selected Hugoniots, LA-4167-MS, Los Alamos Scientific Lab., (1969).

( 8 ) Tanimura, S., Mukai, T., Ishikawa, Y. and Komori, S., Strain Rate and Temperature Effect on Tensile Characteristic of Type 316L Stainless Steels, 248th JEMEKansai Proceedings, (in Japanese), Shinmachi, Nov., (1988), 27-28.

( 9 ) Futakawa, M., Wakui, T., Tanabe, Y. and Ioka, I., Stress-Strain Relationship Evaluation by Inverse Analysis on Indentation Load-Depth Curve, 10th APCNDT Proceedings, Brisbane, Sept., (2001), CD-ROM. 